\title{
Estimating Probabilities of Default for Low Default Portfolios
}

\author{
Katja Pluto and Dirk Tasche*†
}

April 4, 2005

\begin{abstract}
For credit risk management purposes in general, and for allocation of regulatory capital by banks in particular (Basel II), numerical assessments of the credit-worthiness of borrowers are indispensable. These assessments are expressed in terms of probabilities of default (PD) that should incorporate a certain degree of conservatism in order to reflect the prudential risk management style banks are required to apply. In case of credit portfolios that did not at all suffer defaults, or very few defaults only over years, the resulting naive zero or close to zero estimates would clearly not involve such a sufficient conservatism. As an attempt to overcome this issue, we suggest the most prudent estimation principle. This means to estimate the PDs by upper confidence bounds while guaranteeing at the same time a PD ordering that respects the differences in credit quality indicated by the rating grades. The methodology is most easily applied under an assumption of independent default events but can be adapted to the case of correlated defaults.
\end{abstract}

\section{Introduction}

A core input to modern credit risk modeling and managing techniques are probabilities of default (PD) per borrower. As such, the accuracy of the PD estimations determines the quality of the results of credit risk models.

One of the obstacles connected with PD estimations can be the low number of defaults, especially in the better rating grades. Good rating grades might experience many years without any defaults. And even if some defaults occur in a given year, the observed default rates might exhibit a high degree of volatility, due to the relatively low number of borrowers in that grade. But even entire portfolios with low or no defaults are not uncommon in reality. Examples include

\footnotetext{
*Deutsche Bundesbank, Postfach 1006 02, 60006 Frankfurt am Main, Germany E-mail: katja.pluto@gmx.de, dirk.tasche@gmx.net

${ }^{\dagger}$ The opinions expressed in this note are those of the authors and do not necessarily reflect views of the Deutsche Bundesbank.
} 
portfolios with an overall good quality of borrowers (e.g. sovereign or bank portfolios) as well as high-volume-low-number portfolios (e.g. specialized lending).

Usual bank practices for deriving PD values for such exposures often focus on qualitative mapping mechanisms to bank-wide master scales or external ratings. These practices, while widespread in the industry, do not entirely satisfy the desire for a statistical foundation of the assumed PD values. One may "believe" that the PDs per rating grade appear correct, as well as believe that the ordinal ranking and the relative spread between the PDs of two grades is right, but information about the absolute PD figures is lacking. Lastly, it could be questioned whether these rather qualitative methods of $\mathrm{PD}$ calibration fulfill the minimum requirements set out in BCBS (2004a).

The issue has, amongst others, recently been raised in BBA (2004). In that paper, applications of causal default models and of exogenous distribution assumptions on the PDs across the grades have been proposed. In a recent paper, Schuermann and Hanson (2004) present a methodology to estimate PDs by means of migration matrices ("duration method", cf. also Jafry and Schuermann, 2004). This way, non-zero PDs for high-quality rating grades can be estimated more precisely by counting the borrower migrations through the lower grades to eventual default and using Markov chain properties.

This paper focuses on a different issue of PD estimations in low default portfolios. We present a methodology to estimate PDs for portfolios without any defaults, or a very low number of defaults in the overall portfolio. The proposal by Schuermann and Hanson does not provide a solution for such cases, because the duration method requires a certain number of defaults in at least some (usually the low-quality) rating grades.

For estimating PDs, we use all available quantitative information of the rating system and its grades. Moreover, we assume that the ordinal borrower ranking is correct. We do not use any additional assumptions or information. Every additional piece of input would be more on the assumption side, as the low default property of these portfolios does not provide us with more reliable quantitative information.

Our methodology delivers confidence intervals for the PDs of each rating grade. The PD range can be adjusted by the choice of an appropriate confidence level. Moreover, by the most prudent estimation principle our methodology yields monotone PD estimates. We look both at the cases of uncorrelated and correlated default events, in the latter case under assumptions consistent with the Basel risk weight model.

Moreover, we extend the most prudent estimation by two application variants: First we scale our results to overall portfolio central tendencies. Second, we apply our methodology to multi-period data and extend our model by time dependencies of the Basel systematic factor. Both variants should help to align our principle to realistic data sets and to a range of assumptions that can be set according to the specific issues in question when applying our methodology. 
The paper is structured as follows: The two main concepts underlying the methodology - estimating PDs as upper confidence bounds and guaranteeing their monotony by the most prudent estimation principle - are introduced by two examples that assume independence of the default events. The first example deals with a portfolio without any observed defaults. For the second example, we modify the first example by assuming that a few defaults have been observed. In a further section, we show how the methodology can be modified in order to take into account non-zero correlation of default events. This is followed by two sections discussing potential extensions of our methodology, in particular the scaling to the overall portfolio central tendency and an extension of our model to the multi-period case. The last two sections are devoted to discussions of the potential scope of application and of open questions. We conclude with a summary of our proposal. In Appendix $\mathrm{A}$, we provide information on the numerics that is needed to implement the estimation approach we suggest. Appendix B provides additional numerical results to Section 5 ,

\section{Example: No Defaults, Assumption of Independence}

The obligors are distributed to rating grades $A, B$, and $C$, with frequencies $n_{A}, n_{B}$, and $n_{C}$. The grade with the highest credit-worthiness is denoted by $A$, the grade with the lowest creditworthiness is denoted by $C$. Neither in $A$ nor in $B$ nor in $C$ any defaults occurred during the last observation period.

We assume that the - still to be estimated - PDs $p_{A}$ of grade $A, p_{B}$ of grade $B$, and $p_{C}$ of grade $C$ reflect the decreasing credit-worthiness of the grades, in the sense of the following inequality:

$$
p_{A} \leq p_{B} \leq p_{C}
$$

The inequality implies that we assume the ordinal borrower ranking to be correct. According to (2.1), the $\mathrm{PD} p_{A}$ of grade $A$ cannot be greater than the PD $p_{C}$ of grade $C$. As a consequence, the most prudent estimate of the value of $p_{A}$ is obtained under the assumption that the probabilities $p_{A}$ and $p_{C}$ are equal. Then, from (2.1) even follows $p_{A}=p_{B}=p_{C}$. Assuming this relation, we now proceed in determining a confidence region for $p_{A}$ at confidence level $\gamma$. This confidence region $^{1}$ can be described as the set of all admissible values of $p_{A}$ with the property that the probability of not observing any default during the observation period is not less than $1-\gamma$ (for instance for $\gamma=90 \%$ ).

If we have got $p_{A}=p_{B}=p_{C}$, then the three rating grades $A, B$, and $C$ do not differ in their respective riskiness. Hence we have to deal with a homogeneous sample of size $n_{A}+n_{B}+n_{C}$ without any default during the observation period. Assuming unconditional independence of the default events, the probability of observing no defaults turns out to be $\left(1-p_{A}\right)^{n_{A}+n_{B}+n_{C}}$. As a

\footnotetext{
${ }^{1}$ For any value of $p_{A}$ not belonging to this region, the hypothesis that the true $\mathrm{PD}$ takes on this value would have to be rejected at a type I error level of $1-\gamma$.
} 
consequence, we have to solve the inequality

$$
1-\gamma \leq\left(1-p_{A}\right)^{n_{A}+n_{B}+n_{C}}
$$

for $p_{A}$ in order to obtain the confidence region at level $\gamma$ for $p_{A}$ as the set of all the values of $p_{A}$ such that

$$
p_{A} \leq 1-(1-\gamma)^{1 /\left(n_{A}+n_{B}+n_{C}\right)} .
$$

If we choose for the sake of illustration

$$
n_{A}=100, \quad n_{B}=400, \quad n_{C}=300,
$$

Table 1 exhibits some values of confidence levels $\gamma$ with the corresponding maximum values (upper confidence bounds) $\hat{p}_{A}$ of $p_{A}$ such that (2.2a) is still satisfied.

Table 1: Upper confidence bound $\hat{p}_{A}$ of $p_{A}$ as a function of the confidence level $\gamma$. No defaults observed, frequencies of obligors in grades given by (2.3).

\begin{tabular}{c||c|c|c|c|c|c}
$\gamma$ & $50 \%$ & $75 \%$ & $90 \%$ & $95 \%$ & $99 \%$ & $99.9 \%$ \\
\hline$\hat{p}_{A}$ & $0.09 \%$ & $0.17 \%$ & $0.29 \%$ & $0.37 \%$ & $0.57 \%$ & $0.86 \%$
\end{tabular}

According to Table 1 there is a strong dependence of the upper confidence bound $\hat{p}_{A}$ on the confidence level $\gamma$. Intuitively, values of $\gamma$ smaller than $95 \%$ seem more appropriate for estimating the $\mathrm{PD}$ by $\hat{p}_{A}$.

By inequality (2.1), the $\mathrm{PD} p_{B}$ of grade $B$ cannot be greater than the $\mathrm{PD} p_{C}$ of grade $C$ either. Consequently, the most prudent estimate of $p_{B}$ is obtained by assuming $p_{B}=p_{C}$. Assuming additional equality with the $\mathrm{PD} p_{A}$ of the best grade $A$ would violate the most prudent estimation principle, because $p_{A}$ is a lower bound of $p_{B}$. If we have got $p_{B}=p_{C}$, then $B$ and $C$ do not differ in their respective riskiness and may be considered a homogeneous sample of size $n_{B}+n_{C}$. Therefore, the confidence region at level $\gamma$ for $p_{B}$ is obtained from the inequality

$$
1-\gamma \leq\left(1-p_{B}\right)^{n_{B}+n_{C}}
$$

(2.4a) implies that the confidence region for $p_{B}$ consists of all the values of $p_{B}$ that satisfy

$$
p_{B} \leq 1-(1-\gamma)^{1 /\left(n_{B}+n_{C}\right)} .
$$

If we take up again the example described by (2.3), Table 2 exhibits some values of confidence levels $\gamma$ with the corresponding maximum values (upper confidence bounds) $\hat{p}_{B}$ of $p_{B}$ such that (2.4a) is still fulfilled.

For determining the confidence region at level $\gamma$ for $p_{C}$ we only make use of the observations in grade $C$ because by (2.1) there is no obvious upper bound for $p_{C}$. Hence the confidence region at level $\gamma$ for $p_{C}$ consists of those values of $p_{C}$ that satisfy the inequality

$$
1-\gamma \leq\left(1-p_{C}\right)^{n_{C}}
$$


Table 2: Upper confidence bound $\hat{p}_{B}$ of $p_{B}$ as a function of the confidence level $\gamma$. No defaults observed, frequencies of obligors in grades given by (2.3).

\begin{tabular}{c||c|c|c|c|c|c}
$\gamma$ & $50 \%$ & $75 \%$ & $90 \%$ & $95 \%$ & $99 \%$ & $99.9 \%$ \\
\hline$\hat{p}_{B}$ & $0.10 \%$ & $0.20 \%$ & $0.33 \%$ & $0.43 \%$ & $0.66 \%$ & $0.98 \%$
\end{tabular}

Equivalently, the confidence region for $p_{C}$ can be described by

$$
p_{C} \leq 1-(1-\gamma)^{1 / n_{C}} .
$$

Coming back to our example (2.3), Table 3 lists some values of confidence levels $\gamma$ with the corresponding maximum values (upper confidence bounds) $\hat{p}_{C}$ of $p_{C}$ such that (2.5a) is still fulfilled.

Table 3: Upper confidence bound $\hat{p}_{C}$ of $p_{C}$ as a function of the confidence level $\gamma$. No defaults observed, frequencies of obligors in grades given by (2.3).

\begin{tabular}{c||c|c|c|c|c|c}
$\gamma$ & $50 \%$ & $75 \%$ & $90 \%$ & $95 \%$ & $99 \%$ & $99.9 \%$ \\
\hline$\hat{p}_{C}$ & $0.23 \%$ & $0.46 \%$ & $0.76 \%$ & $0.99 \%$ & $1.52 \%$ & $2.28 \%$
\end{tabular}

Comparison of Tables 1, 2] and 3] shows that - besides the confidence level $\gamma$ - the applicable sample size is a main driver of the upper confidence bound. The smaller the sample size that can be made use of, the greater will be the upper confidence bound. This is not an undesirable effect because intuitively the credit-worthiness ought to be the better, the greater is the number of obligors in a portfolio without any default observation.

As the results presented so far seem plausible, we suggest to use upper confidence bounds as described by (2.2b), (2.4b), and (2.5b) as estimates for the PDs in portfolios without observed defaults. The case of three rating grades we have considered in this section can readily be generalized to an arbitrary number of grades. We do not present the details here.

However, the larger the number of obligors in the entire portfolio is, the more often some defaults will occur in some grades at least, even if the general quality of the portfolio is very high. This case is not covered by (2.2b), (2.4b), and (2.5b). In the following section, we will show - still keeping the assumption of independence of the default events - how the most prudent estimation methodology can be adapted to the case of a non-zero but still low number of defaults.

\section{Example: Few Defaults, Assumption of Independence}

We consider again the portfolio from Section 2 with the frequencies $n_{A}, n_{B}$, and $n_{C}$. In contrast to Section 2, this time we assume that during the last period no default was observed in grade $A$, two defaults were observed in grade $B$, and one default was observed in grade $C$. 
As in Section 2. we determine a most prudent confidence region for the $\mathrm{PD} p_{A}$ of $A$. Again, we do so by assuming that the PDs of the three grades are equal. This allows us to treat the entire portfolio as a homogeneous sample of size $n_{A}+n_{B}+n_{C}$. Then the probability of observing not more than three defaults is given by the expression

$$
\sum_{i=0}^{3}\left(\begin{array}{c}
n_{A}+n_{B}+n_{C} \\
i
\end{array}\right) p_{A}^{i}\left(1-p_{A}\right)^{n_{A}+n_{B}+n_{C}-i} .
$$

(3.1) follows from the fact that the number of defaults in the portfolio is binomially distributed as long as the default events are independent. As a consequence of (3.1), the confidence region ${ }^{2}$ at level $\gamma$ for $p_{A}$ is given as the set of all the values of $p_{A}$ that satisfy the inequality

$$
1-\gamma \leq \sum_{i=0}^{3}\left(\begin{array}{c}
n_{A}+n_{B}+n_{C} \\
i
\end{array}\right) p_{A}^{i}\left(1-p_{A}\right)^{n_{A}+n_{B}+n_{C}-i} .
$$

The tail distribution of a binomial distribution can be expressed in terms of an appropriate beta distribution function. Thus, inequality (3.2) can be solved analytically ${ }^{3}$ for $p_{A}$. For details, see Appendix A. If we assume again that the obligors' numbers per grade are as in (2.3), Table 4 shows maximum solutions $\hat{p}_{A}$ of (3.2) for different confidence levels $\gamma$.

Table 4: Upper confidence bound $\hat{p}_{A}$ of $p_{A}$ as a function of the confidence level $\gamma$. No default observed in grade $A$, two defaults observed in grade $B$, one default observed in grade $C$, frequencies of obligors in grades given by (2.3).

\begin{tabular}{c||c|c|c|c|c|c}
$\gamma$ & $50 \%$ & $75 \%$ & $90 \%$ & $95 \%$ & $99 \%$ & $99.9 \%$ \\
\hline$\hat{p}_{A}$ & $0.46 \%$ & $0.65 \%$ & $0.83 \%$ & $0.97 \%$ & $1.25 \%$ & $1.62 \%$
\end{tabular}

Although in grade $A$ no defaults have been observed, the three defaults that occurred during the observation period enter the calculation. They effect the upper confidence bounds, which are much higher than those in Table 1. This is a consequence of the precautionary assumption $p_{A}=p_{B}=p_{C}$. However, if we alternatively considered grade $A$ alone (by reevaluating (2.5b) with $n_{A}=100$ instead of $n_{C}$ ), we would obtain an upper confidence bound $1.38 \%$ at level $\gamma=75 \%$. This value is still much higher than the one that has been calculated under the precautionary assumption $p_{A}=p_{B}=p_{C}-$ a consequence of the low frequency of obligors in grade $A$ in this example. Nevertheless, we see that the methodology described by (3.2) yields fairly reasonable results.

In order to determine the confidence region at level $\gamma$ for $p_{B}$, as in Section 2 we assume that $p_{B}$ takes its greatest possible value according to (2.1), i.e. that we have $p_{B}=p_{C}$. In this situation,

\footnotetext{
${ }^{2}$ We calculate the simple and intuitive exact Clopper-Pearson interval. For an overview of this approach, as well as potential alternatives, see Brown et al. (2001).

${ }^{3}$ Alternatively, solving directly (3.2) for $p_{A}$ by means of numerical tools is not too difficult either (see Appendix $\mathrm{A}$ Proposition A.1 for additional information).
} 
we have got a homogeneous portfolio with $n_{B}+n_{C}$ obligors, PD $p_{B}$, and three observed defaults. In complete analogy to (3.1), the probability of observing no more than three defaults in one period then can be written as

$$
\sum_{i=0}^{3}\left({ }_{i}^{n_{B}+n_{C}}\right) p_{B}^{i}\left(1-p_{B}\right)^{n_{B}+n_{C}-i}
$$

Hence, the confidence region at level $\gamma$ for $p_{B}$ turns out to be the set of all the admissible values of $p_{B}$ which satisfy the inequality

$$
1-\gamma \leq \sum_{i=0}^{3}\left(\begin{array}{c}
n_{B}+n_{C} \\
i
\end{array}\right) p_{B}^{i}\left(1-p_{B}\right)^{n_{B}+n_{C}-i}
$$

By analytically or numerically solving (3.4) for $p_{B}$ - with frequencies of obligors in the grades as in (2.3) - we obtain Table 5 with some maximum solutions $\hat{p}_{B}$ of (3.4) for different confidence levels $\gamma$.

Table 5: Upper confidence bound $\hat{p}_{B}$ of $p_{B}$ as a function of the confidence level $\gamma$. No default observed in grade $A$, two defaults observed in grade $B$, one default observed in grade $C$, frequencies of obligors in grades given by (2.3).

\begin{tabular}{c||c|c|c|c|c|c}
$\gamma$ & $50 \%$ & $75 \%$ & $90 \%$ & $95 \%$ & $99 \%$ & $99.9 \%$ \\
\hline$\hat{p}_{B}$ & $0.52 \%$ & $0.73 \%$ & $0.95 \%$ & $1.10 \%$ & $1.43 \%$ & $1.85 \%$
\end{tabular}

From the given numbers of defaults in the different grades it becomes clear that a stand-alone treatment of grade $B$ would yield still much higher values ${ }^{4}$ for the upper confidence bounds. The upper confidence bound $0.52 \%$ of the confidence region at level $50 \%$ is almost identical with the naive frequency based PD estimate $2 / 400=0.5 \%$ that could alternatively have been calculated for grade $B$ in this example.

For determining the confidence region at level $\gamma$ for the $\mathrm{PD} p_{C}$, by the same rationale as in Section 2 the grade $C$ must be considered a stand-alone portfolio. According to the assumption made in the beginning of this section, one default occurred among the $n_{C}$ obligors in $C$. Hence we see that the confidence region for $p_{C}$ is the set of all admissible values of $p_{C}$ that satisfy the inequality

$$
1-\gamma \leq \sum_{i=0}^{1}\left(\begin{array}{c}
n_{C} \\
i
\end{array}\right) p_{C}^{i}\left(1-p_{C}\right)^{n_{C}-i}=\left(1-p_{C}\right)^{n_{C}}+n_{C} p_{C}\left(1-p_{C}\right)^{n_{C}-1} .
$$

For obligor frequencies as assumed in example (2.3), Table 6 exhibits some maximum solutions ${ }^{5}$ $\hat{p}_{C}$ of (3.5) for different confidence levels $\gamma$.

\footnotetext{
${ }^{4}$ At level $99.9 \%$, e.g., $2.78 \%$ would be the value of the upper confidence bound.

${ }^{5}$ If we had assumed that two defaults occurred in grade $B$ but no default was observed in grade $C$, then we would have obtained smaller upper bounds for $p_{C}$ than for $p_{B}$. As this is not a desirable effect, a possible -
} 
Table 6: Upper confidence bound $\hat{p}_{C}$ of $p_{C}$ as a function of the confidence level $\gamma$. No default observed in grade $A$, two defaults observed in grade $B$, one default observed in grade $C$, frequencies of obligors in grades given by (2.3).

\begin{tabular}{c||c|c|c|c|c|c}
$\gamma$ & $50 \%$ & $75 \%$ & $90 \%$ & $95 \%$ & $99 \%$ & $99.9 \%$ \\
\hline$\hat{p}_{C}$ & $0.56 \%$ & $0.90 \%$ & $1.29 \%$ & $1.57 \%$ & $2.19 \%$ & $3.04 \%$
\end{tabular}

So far, we have described how to generalize the methodology from Section 2 to the case where non-zero default frequencies have been recorded. In the following section we investigate the impact of non-zero default correlation on the PD estimates that are effected by applying the most prudent estimation methodology.

\section{Example: Correlated Default Events}

In this section, we describe the dependence of the default events with the one-factor probit model $^{6}$ that was the starting point for developing the risk weight functions given in BCBS $(2004 a){ }^{7}$. First, we use the example from Section 2 and assume that no default at all was observed in the whole portfolio during the last period. In order to illustrate the effects of correlation, we apply the minimum value of the asset correlation that appears in the Basel II corporate risk weight function. This minimum value is $12 \%$ (BCBS, 2004a, paragraph 272). Our model, however, works with any other correlation assumption as well. Likewise, the most prudent estimation principle could potentially be applied to others than the Basel II type credit risk model as long as the inequalities can be solved for $p_{A}, p_{B}$ and $p_{C}$, respectively.

Under the assumptions of this section, the confidence region at level $\gamma$ for $p_{A}$ is represented as the set of all admissible values of $p_{A}$ that satisfy the inequality (cf. Bluhm et al., 2003, Sections 2.1.2 and 2.5.1 for the derivation)

$$
1-\gamma \leq \int_{-\infty}^{\infty} \varphi(y)\left(1-\Phi\left(\frac{\Phi^{-1}\left(p_{A}\right)-\sqrt{\rho} y}{\sqrt{1-\rho}}\right)\right)^{n_{A}+n_{B}+n_{C}} d y
$$

where $\varphi$ and $\Phi$ stand for the standard normal density and standard normal distribution function, respectively. $\Phi^{-1}$ denotes the inverse function of $\Phi$, and $\rho$ is the asset correlation (here $\rho$ is chosen as $\rho=12 \%$ ). Similarly to (2.2a), the right-hand side of inequality (4.1) tells us the one-period probability of not observing any default among $n_{A}+n_{B}+n_{C}$ obligors with average PD $p_{A}$.

conservative - work-around could be to increment the number of defaults in grade $C$ up to the point where $p_{C}$ would take on a greater value than $p_{B}$. Nevertheless, in this case one would have to make sure that the applied rating system yields indeed a correct ranking of the obligors.

${ }^{6}$ According to De Finetti's theorem (see, e.g., Durrett, 1996, Theorem (6.8)), assuming one systematic factor only is not very restrictive.

${ }^{7}$ See Gordv (2003) and BCBS (2004b) for the background of the risk weight functions. In the case of non-zero realized default rates Balthazar (2004) uses the one-factor model for deriving confidence intervals of the PDs. 
Solving ${ }^{8}$ Equation (4.1) numerically ${ }^{9}$ for the frequencies as given in (2.3) leads to Table 7 with maximum solutions $\hat{p}_{A}$ of (4.1) for different confidence levels $\gamma$.

Table 7: Upper confidence bound $\hat{p}_{A}$ of $p_{A}, \hat{p}_{B}$ of $p_{B}$ and $\hat{p}_{C}$ of $p_{C}$ as a function of the confidence level $\gamma$. No defaults observed, frequencies of obligors in grades given by (2.3) . Case of correlated default events.

\begin{tabular}{c||c|c|c|c|c|c}
$\gamma$ & $50 \%$ & $75 \%$ & $90 \%$ & $95 \%$ & $99 \%$ & $99.9 \%$ \\
\hline$\hat{p}_{A}$ & $0.15 \%$ & $0.40 \%$ & $0.86 \%$ & $1.31 \%$ & $2.65 \%$ & $5.29 \%$ \\
\hline$\hat{p}_{B}$ & $0.17 \%$ & $0.45 \%$ & $0.96 \%$ & $1.45 \%$ & $2.92 \%$ & $5.77 \%$ \\
\hline$\hat{p}_{C}$ & $0.37 \%$ & $0.92 \%$ & $1.89 \%$ & $2.78 \%$ & $5.30 \%$ & $9.84 \%$
\end{tabular}

Comparing the values from the first line of Table 7 with Table 1 shows that the impact of taking care of correlations is moderate for the low confidence levels $50 \%$ and $75 \%$. The impact is much higher for the levels higher than $90 \%$ (for the confidence level $99.9 \%$ the bound is even six times larger). This observation reflects the general fact that introducing unidirectional stochastic dependence in a sum of random variables entails a redistribution of probability mass from the center of the distribution towards its lower and upper limits.

The formulae for the estimations of upper confidence bounds for $p_{B}$ and $p_{C}$ can be derived analogously to (4.1) (in combination with (2.4a) and (2.5a)). This yields the inequalities

$$
1-\gamma \leq \int_{-\infty}^{\infty} \varphi(y)\left(1-\Phi\left(\frac{\Phi^{-1}\left(p_{B}\right)-\sqrt{\rho} y}{\sqrt{1-\rho}}\right)\right)^{n_{B}+n_{C}} d y
$$

and

$$
1-\gamma \leq \int_{-\infty}^{\infty} \varphi(y)\left(1-\Phi\left(\frac{\Phi^{-1}\left(p_{C}\right)-\sqrt{\rho} y}{\sqrt{1-\rho}}\right)\right)^{n_{C}} d y
$$

to be solved for $p_{B}$ and $p_{C}$ respectively. The numerical calculations with (4.2a) and (4.2b) do not deliver additional qualitative insights. For the sake of completeness, however, the maximum solutions $\hat{p}_{B}$ of (4.2a) and $\hat{p}_{C}$ of (4.2b) for different confidence levels $\gamma$ are listed in lines 2 and 3 of Table 7 respectively.

Second, we apply our correlated model to the example from Section [3 and assume that three defaults were observed during the last period. In analogy to Equations (3.1), (3.2) and (4.1), the

\footnotetext{
${ }^{8}$ See Appendix A Proposition A.2 for additional information. Taking into account correlations entails an increase in numerical complexity. Therefore, it might seem to be more efficient to deal with the correlation problem by choosing an appropriately enlarged confidence level in the independent default events approach as described in Sections 2 and 3 However, it remains open how a confidence level for the uncorrelated case, that "appropriately" adjusts for the correlations, can be derived.

${ }^{9}$ The more intricate calculations for this paper were conducted by means of the software $\mathrm{R}$ (cf. R Development Core Team, 2003).
} 
confidence region at level $\gamma$ for $p_{A}$ is represented as the set of all values of $p_{A}$ that satisfy the inequality

$$
1-\gamma \leq \int_{-\infty}^{\infty} \varphi(y) \sum_{i=0}^{3}\left(\begin{array}{c}
n_{A}+n_{B}+n_{C} \\
i
\end{array}\right) G\left(p_{A}, \rho, y\right)^{i}\left(1-G\left(p_{A}, \rho, y\right)\right)^{n_{A}+n_{B}+n_{C}-i} d y,
$$

where the function $G$ is defined by

$$
G(p, \rho, y)=\frac{\Phi^{-1}(p)-\sqrt{\rho} y}{\sqrt{1-\rho}} .
$$

Solving (4.3a for $\hat{p}_{A}$ with obligor frequencies as given in (2.3), and the respective modified equations for $\hat{p}_{B}$ and $\hat{p}_{C}$ yields the following results:

Table 8: Upper confidence bound $\hat{p}_{A}$ of $p_{A}, \hat{p}_{B}$ of $p_{B}$ and $\hat{p}_{C}$ of $p_{C}$ as a function of the confidence level $\gamma$. No default observed in grade A, two defaults observed in grade $B$, one default observed in grade $C$, frequencies of obligors in grades given by (2.3). Case of correlated default events.

\begin{tabular}{c||c|c|c|c|c|c}
$\gamma$ & $50 \%$ & $75 \%$ & $90 \%$ & $95 \%$ & $99 \%$ & $99.9 \%$ \\
\hline$\hat{p}_{A}$ & $0.72 \%$ & $1.42 \%$ & $2.50 \%$ & $3.42 \%$ & $5.88 \%$ & $10.08 \%$ \\
\hline$\hat{p}_{B}$ & $0.81 \%$ & $1.59 \%$ & $2.77 \%$ & $3.77 \%$ & $6.43 \%$ & $10.92 \%$ \\
\hline$\hat{p}_{C}$ & $0.84 \%$ & $1.76 \%$ & $3.19 \%$ & $4.41 \%$ & $7.68 \%$ & $13.14 \%$
\end{tabular}

Not surprisingly, the maximum solutions for $\hat{p}_{A}, \hat{p}_{B}$ and $\hat{p}_{C}$ increase if we introduce defaults in our example. Other than that, the results do not deliver essential additional insights.

\section{Potential Extension: Calibration by Scaling Factors}

One of the drawbacks of the most prudent estimation principle is that in the few defaults case, for all grades the upper confidence bound PD estimates are higher than the average default rate of the overall portfolio. This phenomenon is not surprising, given that we include all defaults of the overall portfolio in the upper confidence bound estimation even for the highest rating grade. However, these estimates might be regarded as too conservative by some practitioners.

A potential remedy would be a scaling ${ }^{10}$ of all of our estimates towards the central tendency (the average portfolio default rate). We introduce a scaling factor $K$ to our estimates such that the overall portfolio default rate is exactly met, i.e.

$$
\frac{\hat{p}_{A} n_{A}+\hat{p}_{B} n_{B}+\hat{p}_{C} n_{C}}{n_{A}+n_{B}+n_{C}} K=P D_{\text {Portfolio }} .
$$

The new, scaled PD estimates will then be

$$
\hat{p}_{X, \text { scaled }}=K \hat{p}_{X}, \quad X=A, B, C .
$$

\footnotetext{
${ }^{10}$ A similar scaling procedure has recently been suggested by Cathcart and Beniamin (2005).
} 
The results of the application of such a scaling factor to our few defaults examples of Sections 3] and 4 are shown in Tables 9 and 10, respectively.

Table 9: Upper confidence bounds $\hat{p}_{A, \text { scaled }}$ of $p_{A}, \hat{p}_{B, \text { scaled }}$ of $p_{B}$ and $\hat{p}_{C, \text { scaled }}$ of $p_{C}$ as a function of the confidence level $\gamma$ after scaling to the central tendency. No default observed in grade $A$, two defaults observed in grade $B$, one default observed in grade $C$, frequencies of obligors in grades given by (2.3). Case of uncorrelated default events.

\begin{tabular}{c||c|c|c|c|c|c}
$\gamma$ & $50 \%$ & $75 \%$ & $90 \%$ & $95 \%$ & $99 \%$ & $99.9 \%$ \\
\hline Central tendency & $0.375 \%$ & $0.375 \%$ & $0.375 \%$ & $0.375 \%$ & $0.375 \%$ & $0.375 \%$ \\
\hline$K$ & 0.71 & 0.48 & 0.35 & 0.30 & 0.22 & 0.17 \\
\hline$\hat{p}_{A, \text { scaled }}$ & $0.33 \%$ & $0.31 \%$ & $0.29 \%$ & $0.29 \%$ & $0.28 \%$ & $0.27 \%$ \\
\hline$\hat{p}_{B, \text { scaled }}$ & $0.37 \%$ & $0.35 \%$ & $0.34 \%$ & $0.33 \%$ & $0.32 \%$ & $0.31 \%$ \\
\hline$\hat{p}_{C, \text { scaled }}$ & $0.40 \%$ & $0.43 \%$ & $0.46 \%$ & $0.47 \%$ & $0.49 \%$ & $0.50 \%$
\end{tabular}

Table 10: Upper confidence bounds $\hat{p}_{A, \text { scaled }}$ of $p_{A}, \hat{p}_{B, \text { scaled }}$ of $p_{B}$ and $\hat{p}_{C, \text { scaled }}$ of $p_{C}$ as a function of the confidence level $\gamma$ after scaling to the central tendency. No default observed in grade $A$, two defaults observed in grade $B$, one default observed in grade $C$, frequencies of obligors in grades given by (2.3). Case of correlated default events.

\begin{tabular}{c||c|c|c|c|c|c}
$\gamma$ & $50 \%$ & $75 \%$ & $90 \%$ & $95 \%$ & $99 \%$ & $99.9 \%$ \\
\hline Central tendency & $0.375 \%$ & $0.375 \%$ & $0.375 \%$ & $0.375 \%$ & $0.375 \%$ & $0.375 \%$ \\
\hline$K$ & 0.46 & 0.23 & 0.13 & 0.09 & 0.05 & 0.03 \\
\hline$\hat{p}_{A, \text { scaled }}$ & $0.33 \%$ & $0.33 \%$ & $0.32 \%$ & $0.32 \%$ & $0.32 \%$ & $0.32 \%$ \\
\hline$\hat{p}_{B, \text { scaled }}$ & $0.38 \%$ & $0.37 \%$ & $0.36 \%$ & $0.36 \%$ & $0.35 \%$ & $0.35 \%$ \\
\hline$\hat{p}_{C \text {,scaled }}$ & $0.39 \%$ & $0.40 \%$ & $0.41 \%$ & $0.42 \%$ & $0.42 \%$ & $0.42 \%$
\end{tabular}

The average estimated portfolio PD will now fit exactly the overall portfolio central tendency. Thus, we loose all conservatism in our estimations. Given the poor default data base in typical applications of our methodology, this might be seen as a disadvantage rather than an advantage. By using the most prudent estimation principle to derive "relative" PDs before scaling them down to the final results, we preserve however the sole dependence of the PD estimates upon the borrower frequencies in the respective rating grades, as well as the monotony of the PDs.

There remains the question of the appropriate confidence level for above calculation. Although the average estimated portfolio PD now always fits the overall portfolio default rate, the confidence level determines the "distribution" of that rate over the rating grades. In above example, though, the differences in distribution appear small, especially in the correlated case, such that 
we would not explore this issue further. The confidence level could, in practice, be used to control for the spread of PD estimates over the rating grades - the higher the confidence level, the higher the spread.

However, above scaling only works if there is a non-zero number of defaults in the overall portfolio. Zero default portfolios would indeed be treated worse if we continue to apply our original proposal to them, compared to using scaled PDs for low default portfolios.

A variant of above scaling proposal, that takes care of both issues, is the use of an upper confidence bound for the overall portfolio PD in lieu of the actual default rate. This upper confidence bound for the overall portfolio $\mathrm{PD}$, incidently, equals the most prudent estimate for the highest rating grade. Then, the same scaling methodology as described above can be applied. The results of its application to the few defaults examples as in Tables 9 and 10 are presented in Tables 11 and 12 .

Table 11: Upper confidence bounds $\hat{p}_{A, \text { scaled }}$ of $p_{A}, \hat{p}_{B, \text { scaled }}$ of $p_{B}$ and $\hat{p}_{C, \text { scaled }}$ of $p_{C}$ as a function of the confidence level $\gamma$ after scaling to the upper confidence bound of the overall portfolio PD. No default observed in grade A, two defaults observed in grade $B$, one default observed in grade $C$, frequencies of obligors in grades given by (2.3). Case of uncorrelated default events.

\begin{tabular}{c||c|c|c|c|c|c}
$\gamma$ & $50 \%$ & $75 \%$ & $90 \%$ & $95 \%$ & $99 \%$ & $99.9 \%$ \\
\hline Upper bound for portfolio PD & $0.46 \%$ & $0.65 \%$ & $0.83 \%$ & $0.97 \%$ & $1.25 \%$ & $1.62 \%$ \\
\hline$K$ & 0.87 & 0.83 & 0.78 & 0.77 & 0.74 & 0.71 \\
\hline$\hat{p}_{A, \text { scaled }}$ & $0.40 \%$ & $0.54 \%$ & $0.65 \%$ & $0.74 \%$ & $0.92 \%$ & $1.16 \%$ \\
\hline$\hat{p}_{B, \text { scaled }}$ & $0.45 \%$ & $0.61 \%$ & $0.74 \%$ & $0.84 \%$ & $1.06 \%$ & $1.32 \%$ \\
\hline$\hat{p}_{C \text {,scaled }}$ & $0.49 \%$ & $0.75 \%$ & $1.01 \%$ & $1.22 \%$ & $1.62 \%$ & $2.17 \%$
\end{tabular}

Table 12: Upper confidence bounds $\hat{p}_{A, \text { scaled }}$ of $p_{A}, \hat{p}_{B, \text { scaled }}$ of $p_{B}$ and $\hat{p}_{C, \text { scaled }}$ of $p_{C}$ as a function of the confidence level $\gamma$ after scaling to the upper confidence bound of the overall portfolio PD. No default observed in grade A, two defaults observed in grade $B$, one default observed in grade $C$, frequencies of obligors in grades given by (2.3). Case of correlated default events.

\begin{tabular}{c||c|c|c|c|c|c}
$\gamma$ & $50 \%$ & $75 \%$ & $90 \%$ & $95 \%$ & $99 \%$ & $99.9 \%$ \\
\hline Upper bound for portfolio PD & $0.71 \%$ & $1.42 \%$ & $2.50 \%$ & $3.42 \%$ & $5.88 \%$ & $10.08 \%$ \\
\hline$K$ & 0.89 & 0.87 & 0.86 & 0.86 & 0.86 & 0.87 \\
\hline$\hat{p}_{A, \text { scaled }}$ & $0.64 \%$ & $1.24 \%$ & $2.16 \%$ & $2.95 \%$ & $5.06 \%$ & $8.72 \%$ \\
\hline$\hat{p}_{B, \text { scaled }}$ & $0.72 \%$ & $1.38 \%$ & $2.39 \%$ & $3.25 \%$ & $5.54 \%$ & $9.54 \%$ \\
\hline$\hat{p}_{C, \text { scaled }}$ & $0.75 \%$ & $1.53 \%$ & $2.76 \%$ & $3.80 \%$ & $6.61 \%$ & $11.37 \%$
\end{tabular}


As, in contrast to the situation of Tables 9 and [10, in Tables 11 and 12 the overall default rate in the portfolio depends on the confidence level, we observe scaled PD estimates for the grades that increase with growing levels. Nevertheless, the scaled PD estimates for the better grades are still considerably lower than the corresponding unscaled estimates from Sections 3 and 4 . respectively. For the sake of comparison, we provide in Annex $\mathbb{B}$ the analogous numerical results for the no default case.

The advantage of this latter variant of the scaling approach is that the degree of conservatism is actively manageable by the appropriate choice of the confidence level for the estimation of the upper confidence bound of the overall portfolio PD. Moreover, it works for both the zero default and the few defaults case, and thus does not produce a structural break between both scenarios. Lastly, the results are less conservative than the ones of our original methodology.

Consequently, we would propose to use the most prudent estimation principle to derive "relative" PDs over the rating grades, and subsequently scale them down according to the upper bound of the overall portfolio $\mathrm{PD}$, which is once more determined by the most prudent estimation principle with an appropriate confidence level.

\section{Potential Extension: The multi-period case}

So far, we have only considered the situation where estimations are carried out on a one year (or one observation period) data sample. In case of a time series with data from several years, the PDs (per rating grade) for the single years could be estimated and could then be used for calculating weighted averages of the PDs in order to make more efficient use of the data. Proceeding this way, however, the interpretation of the estimates as upper confidence bounds at some pre-defined level would be lost.

Alternatively, the data of all years could be pooled and tackled as in the one-year case. When assuming cross-sectional and intertemporal independence of the default events, the methodology as presented in Sections 2 and 3 can be applied to the data pool by replacing the one-year frequency of a grade with the sum of the frequencies of this grade over the years (analogous for the numbers of defaulted obligors). This way, the interpretation of the results as upper confidence bounds as well as the frequency-dependent degree of conservatism of the estimates will be preserved.

However, when turning to the case of default events which are cross-sectionally and intertemporally correlated, pooling does not allow for an adequate modelling. An example would be a portfolio of long-term loans, where in the intertemporal pool every obligor would appear several times. As a consequence, the dependence structure of the pool would have to be specified very carefully, as the structure of correlation over time and of cross-sectional correlation are likely to differ. 
In this section, we present a multi-period extension of the cross-sectional one-factor correlation model that has been introduced in Section 4. We will take the perspective of an observer of a cohort of obligors over a fixed interval of time. The advantage of such a view arises from the possible conceptional separation of time and cross-section effects. Again, we do not present the methodology in full generality but rather introduce it by way of an example.

As in Section 4. we assume that, at the beginning of the observation period, we have got $n_{A}$ obligors in grade $A, n_{B}$ obligors in grade $B$, and $n_{C}$ obligors in grade $C$. In contrast to Section 4. the length of the observation period this time is $T>1$. We consider only the obligors that were present at the beginning of the observation period. Any obligors entering the portfolio afterwards are neglected for the purpose of our estimation exercise. Nevertheless, the number of observed obligors may vary from year to year as soon as any defaults occur.

As in the previous sections, we first consider the estimation of the $\mathrm{PD} p_{A}$ for grade $A$. $\mathrm{PD}$ in this section denotes a long-term average one-year probability of default. Working again with the most prudent estimation principle, we assume that the $\mathrm{PDs} p_{A}, p_{B}$, and $p_{C}$ are equal, i.e. $p_{A}=p_{B}=p_{C}=p$. We assume, in the spirit of Gordv (2003), that a default of obligor $i=1, \ldots, N=n_{A}+n_{B}+n_{C}$ in year $t=1, \ldots, T$ is triggered if the change in value of their assets results in a value lower than some default threshold $c$ as described below (Equation (6.3)). Specifically, if $V_{i, t}$ denotes the change in value of obligor $i$ 's assets, $V_{i, t}$ is given by

$$
V_{i, t}=\sqrt{\rho} S_{t}+\sqrt{1-\rho} \xi_{i, t}
$$

where $\rho$ stands for the asset correlation as introduced in Section $4 S_{t}$ is the realisation of the systematic factor in year $t$, and $\xi_{i, t}$ denotes the idiosyncratic component of the change in value. The cross-sectional dependence of the default events stems from the presence of the systematic factor $S_{t}$ in all the obligors' change in value variables. Obligor $i$ 's default occurs in year $t$ if

$$
V_{i, 1}>c, \ldots, V_{i, t-1}>c, V_{i, t} \leq c
$$

The probability

$$
\mathrm{P}\left[V_{i, t} \leq c\right]=p_{i, t}=p
$$

is the parameter we are interested to estimate: It describes the long-term average one-year probability of default among the obligors that have not defaulted before. The indices $i$ and $t$ at $p_{i, t}$ can be dropped because by the assumptions we are going to specify below $p_{i, t}$ will neither depend on $i$ nor on $t$. To some extent, therefore, $p$ may be considered a through-the-cycle PD.

For the sake of computational feasibility, and in order to keep as close as possible to the Basel II risk weight model, we specify the factor variables $S_{t}, t=1, \ldots, T$, and $\xi_{i, t}, i=1, \ldots, N, t=$ $1, \ldots, T$, as standard normally distributed (cf. Bluhm et al., 2003). Moreover, we assume that the random vector $\left(S_{1}, \ldots, S_{T}\right)$ and the random variables $\xi_{i, t}, i=1, \ldots, N, t=1, \ldots, T$, are independent. As a consequence, from (6.1) follows that the change in value variables $V_{i, t}$ are 
all standard normally distributed. Therefore, (6.3) implies that the default threshold ${ }^{11} c$ is determined by

$$
c=\Phi^{-1}(p),
$$

with $\Phi$ denoting the standard normal distribution function.

While the single components $S_{t}$ of the vector of systematic factors generate the cross-sectional correlation of the default events at time $t$, their intertemporal correlation is effected by the dependence structure of the factors $S_{1}, \ldots, S_{T}$. We further assume that not only the components but also the vector as a whole is normally distributed. Since the components of the vector are standardized, its joint distribution is completely determined by the correlation matrix

$$
\left(\begin{array}{ccccc}
1 & r_{1,2} & r_{1,3} & \cdots & r_{1, T} \\
r_{2,1} & 1 & r_{2,3} & \cdots & r_{2, T} \\
\vdots & & \ddots & & \vdots \\
r_{T-1,1} & \cdots & r_{T-1, T-2} & 1 & r_{T-1, T} \\
r_{T, 1} & \cdots & r_{T, T-2} & r_{T, T-1} & 1
\end{array}\right)
$$

Whereas the cross-sectional correlation within one year is constant for any pair of obligors, empirical observation indicates that the effect of intertemporal correlation becomes weaker with increasing distance in time. We express this distance-dependent behavior ${ }^{12}$ of correlations by setting in (6.5a)

$$
r_{s, t}=\vartheta^{|s-t|}, \quad s, t=1, \ldots, T, s \neq t,
$$

for some appropriate $0<\vartheta<1$ to be specified below.

Let us assume that within the $T$ years observation period $k_{A}$ defaults were observed among the obligors that were initially graded $A, k_{B}$ defaults among the initially graded $B$ obligors and $k_{C}$ defaults among the initially graded $C$ obligors. For the estimation of $p_{A}$ according to the most prudent estimation principle, therefore we have to take into account $k=k_{A}+k_{B}+k_{C}$ defaults among $N$ obligors over $T$ years. For any given confidence level $\gamma$, we have to determine the maximum value $\hat{p}$ of all the parameters $p$ such that the inequality

$$
1-\gamma \leq \mathrm{P}[\text { No more than } k \text { defaults observed }]
$$

is satisfied - note that the right-hand side of (6.6) depends on the one-period probability of default $p$. In order to derive a formulation that is accessible to numerical calculation, we have to rewrite the right-hand side of (6.6).

\footnotetext{
${ }^{11}$ At first sight, the fact that in our model the default threshold is constant over time seems to imply that the model does not reflect the possibility of rating migrations. However, by construction of the model, the conditional default threshold at time $t$ given the value $V_{i, t-1}$ will in general differ from $c$. As we make use of the joint distribution of the $V_{i \pm}$, therefore rating migrations are implicitly taken into account.

${ }^{12}$ Blochwitz et al. (2004) proposed the specification of the intertemporal dependence structure according to (6.5b) for the purpose of default probability estimation.
} 
As the first step we develop an expression for obligor $i$ 's conditional probability to default during the observation period, given a realization of the systematic factors $S_{1}, \ldots, S_{T}$. From (6.1), (6.2), (6.4) and by using of the conditional independence of the $V_{i, 1}, \ldots, V_{i, T}$ given the systematic factors, we obtain

$$
\begin{aligned}
\mathrm{P}\left[\text { Oligor } i \text { defaults } \mid S_{1}, \ldots, S_{T}\right] & =\mathrm{P}\left[\min _{t=1, \ldots, T} V_{i, t} \leq \Phi^{-1}(p) \mid S_{1}, \ldots, S_{T}\right] \\
& =1-\mathrm{P}\left[\xi_{i, 1}>G\left(p, \rho, S_{1}\right), \ldots, \xi_{i, T}>G\left(p, \rho, S_{T}\right) \mid S_{1}, \ldots, S_{T}\right] \\
& =1-\prod_{t=1}^{T}\left(1-\Phi\left(G\left(p, \rho, S_{t}\right)\right)\right),
\end{aligned}
$$

where the function $G$ is defined as in (4.3b). By construction, in our model all the probabilities $\mathrm{P}\left[\right.$ Oligor $i$ defaults $\left.\mid S_{1}, \ldots, S_{T}\right]$ are equal, so that, for any of the $i$ s, we can define

$$
\begin{aligned}
\pi\left(S_{1}, \ldots, S_{T}\right) & =\mathrm{P}\left[\text { Oligor } i \text { defaults } \mid S_{1}, \ldots, S_{T}\right] \\
& =1-\prod_{t=1}^{T}\left(1-\Phi\left(G\left(p, \rho, S_{t}\right)\right)\right) .
\end{aligned}
$$

Using this abbreviation, we can write the right-hand side of (6.6) as

$$
\begin{aligned}
\mathrm{P}[\text { No more than } k \text { defaults observed }] & =\sum_{\ell=0}^{k} \mathrm{E}\left[\mathrm{P}\left[\text { Exactly } \ell \text { obligors default } \mid S_{1}, \ldots, S_{T}\right]\right] \\
& =\sum_{\ell=0}^{k}\left(\begin{array}{c}
N \\
\ell
\end{array}\right) \mathrm{E}\left[\pi\left(S_{1}, \ldots, S_{T}\right)^{\ell}\left(1-\pi\left(S_{1}, \ldots, S_{T}\right)\right)^{N-\ell}\right]
\end{aligned}
$$

The expectations in (6.8b) are expectations with respect to the random vector $\left(S_{1}, \ldots, S_{T}\right)$ and have to be calculated as $T$-dimensional integrals involving the density of the $T$-variate standard normal distribution with correlation matrix given by (6.5a) and (6.5b). When solving (6.6) for $\hat{p}$, we calculated the values of these $T$-dimensional integrals by means of Monte-Carlo simulation, taking advantage of the fact that the term

$$
\sum_{\ell=0}^{k}\left(\begin{array}{c}
N \\
\ell
\end{array}\right) \pi\left(S_{1}, \ldots, S_{T}\right)^{\ell}\left(1-\pi\left(S_{1}, \ldots, S_{T}\right)\right)^{N-\ell}
$$

can efficiently be evaluated by making use of (A.1).

In order to present some numerical results for an illustration of how the model works, we have to fix a time horizon $T$ and values for the cross-sectional correlation $\rho$ and the intertemporal correlation parameter $\vartheta$. We choose $T=5$ as BCBS (2004a) requires the credit institutions to base their PD estimates on a time series with minimum length five years. For $\rho$, we choose $\rho=0.12$ as in Section 4 i.e. again a value suggested by BCBS (2004a). Our feeling is that default events with a five years time distance can be regarded as being nearly independent. Statistically, this statement might be interpreted as something like "the correlation of $S_{1}$ and $S_{5}$ is less than $1 \%$ ". Setting $\vartheta=0.3$, we obtain $\operatorname{corr}\left[S_{1}, S_{5}\right]=\vartheta^{4}=0.81 \%$. Thus, the choice $\vartheta=0.3$ seems to 
be reasonable. Note that our choices of the parameters are purely exemplary, as to some extent choosing the values of the parameters is rather a matter of taste or of decisions depending on the available data or the purpose of the estimations.

Table 13 shows the results of the calculations for the case where no defaults at all were observed during five years in the whole portfolio. The results for all the three grades are summarized in one table. For arriving at these results, (6.6) was first evaluated with $N=n_{A}+n_{B}+n_{C}$, then with $N=n_{B}+n_{C}$, and finally with $N=n_{C}$. In all three cases we set $k=0$ in (6.8b) in order to express that no defaults were observed. Not surprisingly, the calculated confidence bounds are much lower than those presented as in Table 7 demonstrating this way the potentially dramatic effect of exploiting longer observation periods.

Table 13: Upper confidence bounds $\hat{p}_{A}$ of $p_{A}, \hat{p}_{B}$ of $p_{B}$ and $\hat{p}_{C}$ of $p_{C}$ as a function of the confidence level $\gamma$. No defaults during 5 years observed, frequencies of obligors in grades given by (2.3). Case of cross-sectionally and intertemporally correlated default events.

\begin{tabular}{c||c|c|c|c|c|c}
$\gamma$ & $50 \%$ & $75 \%$ & $90 \%$ & $95 \%$ & $99 \%$ & $99.9 \%$ \\
\hline$\hat{p}_{A}$ & $0.03 \%$ & $0.06 \%$ & $0.11 \%$ & $0.16 \%$ & $0.30 \%$ & $0.55 \%$ \\
\hline$\hat{p}_{B}$ & $0.03 \%$ & $0.07 \%$ & $0.13 \%$ & $0.18 \%$ & $0.33 \%$ & $0.62 \%$ \\
\hline$\hat{p}_{C}$ & $0.07 \%$ & $0.14 \%$ & $0.26 \%$ & $0.37 \%$ & $0.67 \%$ & $1.23 \%$
\end{tabular}

For Table 14 we did essentially the same computations as for Table13 the difference being that we assumed that during five years $k_{A}=0$ defaults were observed in grade $A, k_{B}=2$ defaults were observed in grade $B$, and $k_{C}=1$ defaults were observed in grade $C$ (as in Sections 3 and 4 during one year). As a consequence, we had to set $k=3$ in (6.8b) for calculating the upper confidence bounds for $p_{A}$ and $p_{B}$, as well as $k=1$ for the upper confidence bounds of $p_{C}$. Comparing here with the results presented in Table 8] we observe again a very strong effect of taking into account a longer time series.

Table 14: Upper confidence bounds $\hat{p}_{A}$ of $p_{A}, \hat{p}_{B}$ of $p_{B}$ and $\hat{p}_{C}$ of $p_{C}$ as a function of the confidence level $\gamma$. During 5 years no default observed in grade $A$, two defaults observed in grade $B$, and one default observed in grade $C$. Frequencies of obligors in grades given by (2.3). Case of cross-sectionally and intertemporally correlated default events.

\begin{tabular}{c||c|c|c|c|c|c}
$\gamma$ & $50 \%$ & $75 \%$ & $90 \%$ & $95 \%$ & $99 \%$ & $99.9 \%$ \\
\hline$\hat{p}_{A}$ & $0.12 \%$ & $0.21 \%$ & $0.33 \%$ & $0.43 \%$ & $0.70 \%$ & $1.17 \%$ \\
\hline$\hat{p}_{B}$ & $0.14 \%$ & $0.24 \%$ & $0.38 \%$ & $0.49 \%$ & $0.77 \%$ & $1.29 \%$ \\
\hline$\hat{p}_{C}$ & $0.15 \%$ & $0.27 \%$ & $0.46 \%$ & $0.61 \%$ & $1.01 \%$ & $1.70 \%$
\end{tabular}




\section{Potential Applications}

The most prudent estimation methodology described in the previous sections can be used for a range of applications, both in a bank internal context as well as in a Basel II context. In the latter case, it might be of specific importance for portfolios where neither internal nor external default data are sufficient to meet the Basel requirements. A prime example might be Specialized Lending. In these high-volume, low-number and low-default portfolios, internal data are often insufficient for PD estimations per rating grade, and might indeed even be insufficient for central tendency estimations for the entire portfolio (across all rating grades). Moreover, mapping to external ratings - although explicitly allowed in the Basel context and widely used in bank internal applications - might be impossible due to the low number of externally rated exposures.

The (conservative) principle of the most prudent estimation could potentially serve as an alternative to the Basel slotting approach, subject to supervisory approval. In this context, the proposed methodology might be interpreted as a specific form of the Basel requirement of conservative estimations in case of data scarcity.

In a wider, bank internal context, the methodology might be used for all sorts of low default portfolios. In particular, it could serve as a complement to other estimation methods, whether

this be mapping to external ratings, the proposals by Schuermann and Hanson (2004) or others. As such, we see our proposed methodology as one additional source for PD calibrations, that should neither invalidate nor prejudge a bank's internal choice of calibration methodologies.

However, we tend to believe that our proposed methodology should only be applied to whole rating systems and portfolios. The - at first sight imaginable - calibration of PDs of individual, low default rating grades by the most prudent estimation principle within an otherwise data rich portfolio seems infeasible because of the unavoidable structural break between average PDs (data rich rating grades) and upper PD bounds (low default rating grades). Similarly, we believe that the application of the methodology for back-testing or similar validation tools would not add much additional information, as the (e.g. purely expert based) average PDs per rating grade would normally be well below our proposed quantitative upper bounds.

\section{Open Issues}

For potential applications, a number of issues would need to be addressed. In the following, we list the ones that seem to be the most important to us:

- Which confidence levels are appropriate? The proposed most prudent estimate could serve as a conservative proxy for average PDs. In determining the confidence level, the impact of a potential underestimation of these average PDs should be taken into account. One might 
think that the transformation of average PDs into some kind of "stress" PDs, as done in the Basel II and many other credit risk models, could justify rather low confidence levels for the PD estimation in the first place (i.e. using the models as providers of additional buffers against uncertainty). However, this conclusion would be misleading, as it mixes two different types of "stresses": the Basel II model "stress" of the single systematic factor over time, and the estimation uncertainty "stress" of the PD estimations.

Nevertheless, we would argue for moderate confidence levels when applying the most prudent estimation principle, but according to another reasoning: The most common alternative to our methodology, namely deriving PDs from averages of historical default rates per rating grade, yields a comparable probability of underestimating the true PD. As such, high confidence levels in our methodology would be hard to justify.

- At which number of defaults should one deviate from our methodology and use "normal" average PD estimation methods (at least for the overall portfolio central tendency)? Can this critical number be analytically determined?

- If the relative number of defaults in one of the better ratings grades is significantly higher than those in lower rating grades (and within low default portfolios, this might happen with only one or two additional defaults), then our PD estimates can turn out to be nonmonotone. In which cases should this be taken as an indication for the non-correctness of the ordinal ranking? Certainly, monotony or non-monotony of our upper PD bounds do not immediately imply that the average PDs are monotone or non-monotone. Under which conditions would there be statistical evidence of a violation of the monotony requirement for the PDs?

Currently, we do not have definite solutions to above issues. We believe, though, that some of them will involve a certain amount of expert judgment rather than analytical solutions. In particular, that might be the case with the first item. If our proposed approach would be used in a supervisory - say Basel II - context, supervisors might want to think about suitable confidence levels that should be consistently applied.

\section{Conclusions}

In this article, we have introduced a methodology for estimating probabilities of default in low or no default portfolios. The methodology is based on upper confidence intervals by use of the most prudent estimation. Our methodology uses all available quantitative information. In the extreme case of no defaults in the entire portfolio, this information consists solely of the absolute numbers of counter-parties per rating grade.

The lack of defaults in the entire portfolio prevents reliable quantitative statements on both the absolute level of average PDs per rating grade as well as on the relative risk increase from 
rating grade to rating grade. Within the most prudent estimation methodology, we do not use such information. The only additional assumption used is the ordinal ranking of the borrowers, which is assumed to be correct.

Our PD estimates might seem rather high at first sight. However, given the amount of information that is actually available, the results do not appear out of range. We believe that the choice of moderate confidence levels is appropriate within most applications. The results can be scaled to any appropriate central tendency. Additionally, the multi-year context as described in Section [6] might provide further insight.

Acknowledgment. The authors thank Til Schuermann, Claudia Sand, and two anonymous referees for providing useful hints on an earlier draft of this paper.

\section{References}

Balthazar, L. (2004) PD estimates for Basel II. Risk April, 84-85.

Basel Committee on Banking Supervision (BCBS) (2004A) Basel II: International Convergence of Capital Measurement and Capital Standards: a Revised Framework. http://www.bis.org/publ/bcbs107.htm

Basel Committee on Banking Supervision (BCBS) (2004B) An Explanatory Note on the Basel II IRB Risk Weight Functions. http://www.banque-france.fr/gb/supervi/supervi_banc/page3.htm

British Bankers' Association (BBA), London Investment Banking Association (LIBA) and International Swaps and Derivatives Association (ISDA) (2004) The IRB Approach for Low Default Portfolios (LDPs) - Recommendations of the Joint BBA, LIBA, ISDA Industry Working Group. Discussion paper. http://www.isda.org/speeches/pdf/ISDA-LIBA-BBA-LowDefaulPortfolioPaper080904-paper.pdf

Blochwitz, S., Hohl, S., Tasche, D. And Wehn, C. (2004) Validating Default Probabilities on Short Time Series. Capital $\& 5$ Market Risk Insights (Federal Reserve Bank of Chicago), December. http://www.chicagofed.org/banking_information/capital_and_market_risk_insights.cfm

Bluhm, C., Overbeck, L. And C. Wagner (2003) An Introduction to Credit Risk Modeling. Boca Raton: Chapman \& Hall/CRC.

Brown, L., Cai, T. And A. Dasgupta (2001) Interval Estimation for a Binomial Proportion. Statistical Science 16(2), 101-133.

Cathcart, A. and N. Benjamin (2005) Low Default Portfolios: A Proposal for conservative PD estimation. Discussion paper, Financial Services Authority. 
Durrett, R. (1996) Probability: Theory and Examples. Second Edition. Belmont: Wadsworth.

Gordy, M. (2003) A Risk-Factor Model Foundation for Ratings-Based Bank Capital Rules. Journal of Financial Intermediation 12(3), 199-232.

Hinderen, K. (1980) Grundbegriffe der Wahrscheinlichkeitstheorie. Zweiter korrigierter Nachdruck der ersten Auflage. Berlin: Springer-Verlag.

JAFRY, Y. AND T. SchUermann (2004) Measurement, estimation and comparison of credit migration matrices. Journal of Banking $\&$ Finance 28, 2603-2639.

R Development Core Team (2003) R: A language and environment for statistical computing. R Foundation for Statistical Computing, Vienna. http://www.R-project.org

Schuermann, T. and S. Hanson (2004) Estimating Probabilities of Default. Staff Report no. 190, Federal Reserve Bank of New York.

VAsiceK, O. (1997) The loan loss distribution. Working paper, KMV Corporation.

\section{A Appendix}

This appendix provides some additional information on the analytical and numerical solutions of Equations (3.2) and (4.1).

Analytical solution of Equation (3.2). If $X$ is a binomially distributed random variable with size parameter $n$ and success probability $p$, then for any integer $0 \leq k \leq n$ we have

$$
\sum_{i=0}^{k}\left(\begin{array}{c}
n \\
i
\end{array}\right) p^{i}(1-p)^{n-i}=\mathrm{P}[X \leq k]=1-\mathrm{P}[Y \leq p]=\frac{\int_{p}^{1} t^{k}(1-t)^{n-k-1} d t}{\int_{0}^{1} t^{k}(1-t)^{n-k-1} d t}
$$

with Y denoting a beta distributed random variable with parameters $\alpha=k+1$ and $\beta=n-k$

(see, e.g., Hinderer, 1980, Lemma 11.2). The beta distribution function and its inverse function are available in standard numerical tools, e.g. in Excel.

Direct numerical solution of Equation (3.2). The following proposition shows the existence and uniqueness of the solution of (3.2), and, at the same time, provides us with initial values for the numerical root-finding (see (A.2C)).

Proposition A.1 Let $0 \leq k<n$ be integers, and define the function $f_{n, k}:(0,1) \rightarrow \mathbb{R}$ by

$$
f_{n, k}(p)=\sum_{i=0}^{k}\left(\begin{array}{c}
n \\
i
\end{array}\right) p^{i}(1-p)^{n-i}, \quad p \in(0,1) .
$$

Fix some $0<v<1$. Then the equation

$$
f_{n, k}(p)=v
$$


has exactly one solution $0<p=p(v)<1$. Moreover, this solution $p(v)$ satisfies the inequalities

$$
1-\sqrt[n]{v} \leq p(v) \leq \sqrt[n]{1-v}
$$

Proof. A straight-forward calculation yields

$$
\frac{d f_{n, k}(p)}{d p}=-(n-k)\left(\begin{array}{c}
n \\
k
\end{array}\right) p^{k}(1-p)^{n-k-1} .
$$

Hence $f_{n, k}$ is strictly decreasing. This implies uniqueness of the solution of (A.2b). The inequalities

$$
f_{n, 0}(p) \leq f_{n, k}(p) \leq f_{n, n-1}(p)
$$

imply existence of a solution of (A.2b) and the inequalities (A.2C).

Numerical solution of Equation (4.1). For (4.1) we can derive a result similar to Proposition A.1. However, there does not exist an obvious upper bound to the solution $p(v)$ of (A.5b) as in (A.2C).

Proposition A.2 For any probability $0<p<1$, any correlation $0<\rho<1$ and any real number $y$ define

$$
F_{\rho}(p, y)=\Phi\left(\frac{\Phi^{-1}(p)+\sqrt{\rho} y}{\sqrt{1-\rho}}\right)
$$

where we make use of the same notations as for Equation (4.1). Fix a value $0<v<1$ and a positive integer $n$. Then the equation

$$
v=\int_{-\infty}^{\infty} \varphi(y)\left(1-F_{\rho}(p, y)\right)^{n} d y
$$

with $\varphi$ denoting the standard normal density, has exactly one solution $0<p=p(v)<1$. This solution $p(v)$ satisfies the inequality

$$
p(v) \geq 1-\sqrt[n]{v}
$$

Table 15: Upper confidence bounds $\hat{p}_{A, \text { scaled }}$ of $p_{A}, \hat{p}_{B, \text { scaled }}$ of $p_{B}$ and $\hat{p}_{C, \text { scaled }}$ of $p_{C}$ as a function of the confidence level $\gamma$ after scaling to the upper confidence bound of the overall portfolio PD. No default observed, frequencies of obligors in grades given by (2.3). Case of uncorrelated default events.

\begin{tabular}{c||c|c|c|c|c|c}
$\gamma$ & $50 \%$ & $75 \%$ & $90 \%$ & $95 \%$ & $99 \%$ & $99.9 \%$ \\
\hline Upper bound for portfolio PD & $0.09 \%$ & $0.17 \%$ & $0.29 \%$ & $0.37 \%$ & $0.57 \%$ & $0.86 \%$ \\
\hline$K$ & 0.61 & 0.66 & 0.60 & 0.58 & 0.59 & 0.59 \\
\hline$\hat{p}_{A, \text { scaled }}$ & $0.05 \%$ & $0.11 \%$ & $0.17 \%$ & $0.22 \%$ & $0.33 \%$ & $0.51 \%$ \\
\hline$\hat{p}_{B, \text { scaled }}$ & $0.06 \%$ & $0.13 \%$ & $0.20 \%$ & $0.25 \%$ & $0.39 \%$ & $0.58 \%$ \\
\hline$\hat{p}_{C, \text { scaled }}$ & $0.14 \%$ & $0.24 \%$ & $0.45 \%$ & $0.58 \%$ & $0.89 \%$ & $1.35 \%$
\end{tabular}


Proof of Proposition A.1. Note that - for fixed $\rho$ and $y$ - the function $F_{\rho}(p, y)$ is strictly increasing and continuous in $p$. Moreover, we have

$$
0=\lim _{p \rightarrow 0} F_{\rho}(p, y) \quad \text { and } \quad 1=\lim _{p \rightarrow 1} F_{\rho}(p, y)
$$

Equation (A.6) implies existence and uniqueness of the solution of (A.5b).

Define the random variable $Z$ by

$$
Z=F_{\rho}(p, Y),
$$

where $Y$ denotes a standard normally distributed random variable. Then $Z$ has the well-known Vasicek distribution (cf. Vasicek, 1997), and in particular we have

$$
\mathrm{E}[Z]=p
$$

Using (A.7), Equation (A.5b) can be rewritten as

$$
v=\mathrm{E}\left[(1-Z)^{n}\right]
$$

Since $y \mapsto(1-y)^{n}$ is convex for $0<y<1$, by (A.8) Jensen's inequality implies

$$
v=\mathrm{E}\left[(1-Z)^{n}\right] \geq(1-p)^{n} .
$$

As the right-hand side of (A.5b) is decreasing in $p$, (A.5c) now follows from (A.10).

\section{B Appendix}

This appendix provides additional numerical results for the "scaling" extension of the most prudent estimation principle according to Section 5 in the case of no default portfolios. In the examples presented in Tables [15] and 16, the confidence level for deriving the upper confidence bound for the overall portfolio PD, and the confidence levels for the most prudent estimates of

Table 16: Upper confidence bounds $\hat{p}_{A, \text { scaled }}$ of $p_{A}, \hat{p}_{B, \text { scaled }}$ of $p_{B}$ and $\hat{p}_{C, \text { scaled }}$ of $p_{C}$ as a function of the confidence level $\gamma$ after scaling to the upper confidence bound of the overall portfolio PD. No default observed, frequencies of obligors in grades given by (2.3). Case of correlated default events.

\begin{tabular}{c||c|c|c|c|c|c}
$\gamma$ & $50 \%$ & $75 \%$ & $90 \%$ & $95 \%$ & $99 \%$ & $99.9 \%$ \\
\hline Upper bound for portfolio PD & $0.15 \%$ & $0.40 \%$ & $0.86 \%$ & $1.31 \%$ & $2.65 \%$ & $5.29 \%$ \\
\hline$K$ & 0.62 & 0.65 & 0.66 & 0.68 & 0.70 & 0.73 \\
\hline$\hat{p}_{A, \text { scaled }}$ & $0.09 \%$ & $0.26 \%$ & $0.57 \%$ & $0.89 \%$ & $1.86 \%$ & $3.87 \%$ \\
\hline$\hat{p}_{B \text {,scaled }}$ & $0.11 \%$ & $0.29 \%$ & $0.64 \%$ & $0.98 \%$ & $2.05 \%$ & $4.22 \%$ \\
\hline$\hat{p}_{C \text {,scaled }}$ & $0.23 \%$ & $0.59 \%$ & $1.25 \%$ & $1.89 \%$ & $3.72 \%$ & $7.19 \%$
\end{tabular}


PDs per rating grade have always been set equal. Moreover, our methodology always provides equality between the upper bound of the overall portfolio PD and the most prudent estimate for $p_{A}$ according to the respective examples of Sections 2$]$ and 4 